\title{
Homotopy Analysis Method for the Time-Fractional Boussinesq Equation
}

\author{
He Yang ${ }^{1}$ \\ ${ }^{1}$ Department of Mathematics, Augusta University, Augusta, GA 30912, USA
}

\section{Article Info}

Keywords: Homotopy analysis method, Time-fractional Boussinesq equation

2010 AMS: 26A33, 35A35, 34A08

Received: 18 October 2019

Accepted: 10 February 2020

Available online: 25 February 2020

\begin{abstract}
In this paper, the exact and approximate analytical solutions to the time-fractional Boussinesq equation are constructed using the homotopy analysis method. Several examples about the fourth-order and sixth-order time-fractional Boussinesq equations show the flexibility and efficiency of the method. Furthermore, by choosing an appropriate value for the auxiliary parameter $h$, we can obtain the $N$-term approximate solution with improved accuracy.
\end{abstract}

\section{Introduction}

In recent years, the time-fractional differential equations have attracted a large amount of attention due to their broad applications in physics, biology, hydrology and engineering [9]. In particular, the time-fractional Boussinesq equation, as a generalization of the Boussinesq equation, can be used to describe the surface water waves with a long memory property. Roughly speaking, two types of methods have been used to solve the fractional differential equation, including analytical [5,6,12,13,16,18-20] and numerical methods [1,3,7,8,11,14,17,21-24]. As far as the analytical methods for the time-fractional Boussinesq equations are concerned, the authors in [5] applied the modified Kudryashov method to solve the nonlinear conformable time-fractional Boussinesq equations. The authors in [6] used the fractional Lie group method to solve the time-fractional Boussinesq equation. Xu et al. [16] also proposed an iterative method to construct the analytical solution. Recently, a Fourier spectral method [24] was developed to obtain the numerical solutions of the equation.

In this paper, we consider the time-fractional Boussinesq equation, which is defined by replacing the integer-order time derivatives with the fractional-order time derivatives. The fractional derivatives are in the Caputo's sense, so that the same initial conditions for the Boussinesq equation with integer-order derivatives can be imposed for the time-fractional equation. Furthermore, we apply the homotopy analysis method [10] to obtain the exact and approximate solution of the time-fractional Boussinesq equation. By solving various examples using the homotopy analysis method, we can show the flexibility and efficiency of the method. A key component of the method is the selection of the auxiliary parameter $h$. Numerical results provide some insights on how to choose $h$ to obtain the $N$-term approximate solutions with improved accuracy.

The remaining of the paper is as follows: in section 2, we introduce the notations and basic properties of the fractional calculus, and describe the homotopy analysis method for the general nonlinear partial differential equations. In section 3 , several examples about the fourth-order and sixth-order time-fractional Boussinesq equations are presented to demonstrate the performance of the homotopy analysis method. Numerical results about the $N$-term approximate solutions with various $h$ and $\alpha$ are also discussed to provide the guideline of choosing the parameters.

\section{Homotopy Analysis Method}

In this section, we introduce the basic properties of fractional calculus and the homotopy analysis method for the general nonlinear partial differential equations. 


\subsection{Preliminaries and Notations}

In the literature, there are many definitions of fractional derivatives, including the Riemann-Liouville, Caputo, Grünwald-Letnikov, CaputoFabrizio and Atangana-Baleanu derivative $[2,4,15]$. Among all the aforementioned definitions, the Caputo derivative is one of the most widely used definitions for the time-fractional partial differential equations models. It is defined based on the Riemann-Liouville fractional integral. Let $\alpha>0$ and $n$ be the smallest integer that is greater than or equal to $\alpha$, then for any locally integrable function $f$, its Riemann-Liouville (RL) fractional integral of order $\alpha$ is given by

$$
J^{\alpha} f(t)=\frac{1}{\Gamma(\alpha)} \int_{0}^{t}(t-s)^{\alpha-1} f(s) d s .
$$

Here $\Gamma$ is the Gamma function. The RL fractional integral (2.1) is a generalization of the $n$-fold integral of $f$. For $f(t)=t^{\beta}$ with $\beta \geq 0$, we can show that $J^{\alpha} t^{\beta}=\frac{\Gamma(\beta+1)}{\Gamma(\alpha+\beta+1)} t^{\alpha+\beta}$. The $\alpha^{\text {th }}$-order Caputo fractional derivative of smooth enough $f$ can then be defined as ${ }_{0} D_{t}^{\alpha} f(t):=J^{n-\alpha}\left(f^{(n)}(t)\right)$, where $f^{(n)}(t)$ is the $n^{\text {th }}$ order derivative of $f$ with respect to $t$ and $\alpha \in(n-1, n]$. That is,

$$
{ }_{0} D_{t}^{\alpha} f(t)=\frac{1}{\Gamma(n-\alpha)} \int_{0}^{t}(t-s)^{n-1-\alpha} f^{(n)}(s) d s .
$$

The Caputo fractional derivative and the Riemann-Liouville fractional integral satisfy the following equality for smooth enough function $f$ :

$$
J^{\alpha}\left({ }_{0} D_{t}^{\alpha} f(t)\right)=f(t)-\sum_{m=0}^{n-1} f^{(m)}(0+) \frac{t^{m}}{m !}, \quad t>0,
$$

where $f^{(m)}(0+)$ is the right-hand limit of $f^{(m)}(x)$ when $x$ approaches zero from the right. Some exact solutions of the time-fractional differential equations can be represented using the Mittag-Leffler function [15], which is defined by the following series

$$
E_{\alpha}(t)=\sum_{m=0}^{\infty} \frac{t^{m}}{\Gamma(1+m \alpha)}, \quad \text { for } \alpha>0 .
$$

\subsection{Homotopy Analysis Method}

Suppose we consider the nonlinear partial differential equation

$$
\mathscr{N}(u(x, t))=0,
$$

where $\mathscr{N}$ in general is a nonlinear operator, and $u(x, t)$ is the exact solution to the equation (2.5). Let $\mathscr{L}$ be a linear operator that is a part of $\mathscr{N}$, then we consider the following equation

$$
(1-q) \mathscr{L}\left(u(x, t)-u_{0}(x, t)\right)=q h \mathscr{N}(u(x, t)) .
$$

Here $q \in[0,1], h$ is a non-zero auxiliary parameter and $u_{0}(x, t)$ is an initial guess of the solution. We can see that when $q=0$, equation (2.6) becomes $\mathscr{L}\left(u(x, t)-u_{0}(x, t)\right)=0$. Therefore, $u_{0}(x, t)$ is the solution to (2.6) when $q=0$, and $u(x, t)$ is the solution when $q=1$. Since there is a solution to equation (2.6) for every given value of $q \in[0,1]$, we can replace $u(x, t)$ in (2.6) with $U(x, t ; q)$. That is,

$$
(1-q) \mathscr{L}\left(U(x, t ; q)-u_{0}(x, t)\right)=q h \mathscr{N}(U(x, t ; q)) .
$$

We further assume that $U(x, t ; q)$ can be written as $U(x, t ; q)=u_{0}(x, t)+\sum_{m=1}^{\infty} u_{m}(x, t) q^{m}$, and we also assume that all the series are convergent. Thus (2.7) leads to

$$
(1-q) \mathscr{L}\left(\sum_{m=1}^{\infty} u_{m}(x, t) q^{m}\right)=q h \mathscr{N}\left(u_{0}(x, t)+\sum_{m=1}^{\infty} u_{m}(x, t) q^{m}\right) .
$$

We then use the Taylor expansion of equation (2.8) and let the corresponding coefficients of $q^{m}$ on both sides of the equation to be the same for all $m \geq 1$, and get

$$
\begin{aligned}
& \mathscr{L}\left(u_{1}(x, t)\right)=h \mathscr{N}\left(u_{0}(x, t)\right), \\
& \mathscr{L}\left(u_{m}(x, t)-u_{m-1}(x, t)\right)=\left.h \frac{1}{(m-1) !} \frac{\partial^{m-1} \mathscr{N}\left(u_{0}(x, t)\right)}{\partial q^{m-1}}\right|_{q=0}, \quad m \geq 2 .
\end{aligned}
$$

If one can apply the inverse operator of $\mathscr{L}$ (denoted by $\mathscr{L}^{-1}$ ) on both sides of (2.9) and (2.10), then $u_{m}(x, t)$ can be calculated iteratively for $m \geq 1$. Finally, the analytical solution of equation (2.5) can be represented by

$$
U(x, t ; 1)=\sum_{m=0}^{\infty} u_{m}(x, t)
$$

assuming the series above converges.

\section{Application to the Time-Fractional Boussinesq Equation}

In this section, we construct the analytical and approximate analytical solutions of the time-fractional Boussinesq equation using the homotopy analysis method. 


\subsection{Example 1}

We consider the following fourth-order time-fractional Boussinesq equation

$$
\left\{\begin{array}{l}
{ }_{0} D_{t}^{\alpha} u=\beta u_{x x x x}+\gamma u_{x x}+\theta\left(u^{2}\right)_{x x}-4 \theta u^{2}, \quad-\infty<x<\infty, t>0, \\
u(x, 0)=e^{x}, \quad u_{t}(x, 0)=0,
\end{array}\right.
$$

where $\alpha \in(1,2], u$ is a function of $x$ and $t$, the coefficients $\beta>0, \gamma>0$ and $\theta$ are constants. To solve the initial value problem using the homotopy analysis method, we let $\mathscr{L}(u(x, t))={ }_{0} D_{t}^{\alpha} u$ and $\mathscr{N}(u(x, t))={ }_{0} D_{t}^{\alpha} u-\beta u_{x x x x}-\gamma u_{x x}-\theta\left(u^{2}\right)_{x x}+4 \theta u^{2}$ for sufficiently smooth $u(x, t)$. We apply the homotopy analysis method, and let $u_{0}=e^{x}$. Thus (2.9) leads to

$$
{ }_{0} D_{t}^{\alpha} u_{1}=h\left({ }_{0} D_{t}^{\alpha} u_{0}-\beta\left(u_{0}\right)_{x x x x}-\gamma\left(u_{0}\right)_{x x}-\theta\left(\left(u_{0}\right)^{2}\right){ }_{x x}+4 \theta\left(u_{0}\right)^{2}\right)=-h(\beta+\gamma) e^{x} .
$$

We then apply $J^{\alpha}$ on both sides of (3.1) to get

$$
u_{1}(x, t)=-\frac{\beta+\gamma}{\Gamma(1+\alpha)} h e^{x} t^{\alpha}
$$

Similarly, (2.10) leads to

$$
{ }_{0} D_{t}^{\alpha}\left(u_{2}-u_{1}\right)=h\left({ }_{0} D_{t}^{\alpha} u_{1}\right)-h \beta\left(u_{1}\right)_{x x x x}-h \gamma\left(u_{1}\right)_{x x}-h \theta \sum_{j=0}^{1}\left(u_{j} u_{1-j}\right)_{x x}+4 h \theta \sum_{j=0}^{1} u_{j} u_{1-j}=h_{0} D_{t}^{\alpha} u_{1}+\frac{(\beta+\gamma)^{2}}{\Gamma(1+\alpha)} h^{2} e^{x} t^{\alpha} .
$$

Again we apply $J^{\alpha}$ to (3.3) to get

$$
u_{2}(x, t)=-\frac{\beta+\gamma}{\Gamma(1+\alpha)} h(1+h) e^{x} t^{\alpha}+\frac{(\beta+\gamma)^{2}}{\Gamma(1+2 \alpha)} h^{2} e^{x} t^{2 \alpha} .
$$

For general $m \geq 1$, we can obtain

$$
u_{m}(x, t)=(1+h) u_{m-1}-h \beta J^{\alpha}\left(\left(u_{m-1}\right)_{x x x x}\right)-h \gamma J^{\alpha}\left(\left(u_{m-1}\right)_{x x}\right)-h \theta J^{\alpha}\left(\sum_{j=0}^{m-1}\left(u_{j} u_{m-1-j}\right)_{x x}\right)+4 h \theta J^{\alpha}\left(\sum_{j=0}^{m-1} u_{j} u_{m-1-j}\right) .
$$

Using mathematical induction, we can show that

$$
u_{m}(x, t)=e^{x} \sum_{j=0}^{m-1}(-1)^{j+1}\left(\begin{array}{c}
m-1 \\
j
\end{array}\right) \frac{h^{j+1}(1+h)^{m-1-j}}{\Gamma(1+\alpha+j \alpha)} t^{(j+1) \alpha}(\beta+\gamma)^{j+1} .
$$

Therefore, the analytical solution can be represented by

$$
u(x, t)=e^{x}+e^{x} \sum_{m=1}^{\infty} \sum_{j=0}^{m-1}(-1)^{j+1}\left(\begin{array}{c}
m-1 \\
j
\end{array}\right) \frac{h^{j+1}(1+h)^{m-1-j}}{\Gamma(1+\alpha+j \alpha)} t^{(j+1) \alpha}(\beta+\gamma)^{j+1} .
$$

When $h=-1$, equation (3.7) becomes $u(x, t)=e^{x} \sum_{m=0}^{\infty} \frac{t^{m \alpha}(\beta+\gamma)^{m}}{\Gamma(1+m \alpha)}$, which is convergent for all $\beta, \gamma>0, \alpha \in(1,2], x \in \mathbf{R}$ and $t \geq 0$. Other choices of the parameter $h$ will determine the rate of convergence. The $N^{\text {th }}$ order approximation using the homotopy analysis method is given by

$$
U^{N}(x, t)=e^{x}+e^{x} \sum_{m=1}^{N} \sum_{j=0}^{m-1}(-1)^{j+1}\left(\begin{array}{c}
m-1 \\
j
\end{array}\right) \frac{h^{j+1}(1+h)^{m-1-j}}{\Gamma(1+\alpha+j \alpha)} t^{(j+1) \alpha}(\beta+\gamma)^{j+1} .
$$

To investigate the convergence of the approximation solution for various values of $h$, we compute the $L^{\infty}$ error for $x \in[-1,1]$ at $t=1$ and it is shown in Table 1.

\begin{tabular}{crrrrr}
\hline$N$ & $h=-1.2$ & $h=-1.1053$ & $h=-1$ & $h=-0.8$ & $h=-0.6$ \\
\hline 4 & $1.0005 \mathrm{e}-02$ & $1.4127 \mathrm{e}-06$ & $6.7082 \mathrm{e}-03$ & $1.7332 \mathrm{e}-01$ & $7.0676 \mathrm{e}-01$ \\
8 & $1.4976 \mathrm{e}-05$ & $9.8654 \mathrm{e}-08$ & $6.2465 \mathrm{e}-08$ & $1.7657 \mathrm{e}-03$ & $5.2175 \mathrm{e}-02$ \\
12 & $1.1419 \mathrm{e}-08$ & $2.3393 \mathrm{e}-11$ & $4.2633 \mathrm{e}-14$ & $1.2178 \mathrm{e}-05$ & $3.1777 \mathrm{e}-03$ \\
16 & $8.0469 \mathrm{e}-11$ & $1.0658 \mathrm{e}-14$ & 0 & $6.7143 \mathrm{e}-08$ & $1.7199 \mathrm{e}-04$ \\
20 & $6.9278 \mathrm{e}-14$ & $3.5527 \mathrm{e}-15$ & 0 & $3.1885 \mathrm{e}-10$ & $8.5779 \mathrm{e}-06$ \\
\hline
\end{tabular}

Table 1: $\left\|U^{N}(\cdot, T)-u_{\text {exact }}(\cdot, T)\right\|_{\infty, x \in[-1,1]}$ at $T=1$ for various approximation order $N$ and auxiliary parameter $h$ when $\alpha=1.5$.

We can see from Table 1 that at $T=1, h=-1.1053$ leads to the most accurate solution (with the $L^{\infty}$ error being $1.4127 \times 10^{-6}$ ) among all the 4-term approximations, which implies that the 4-term approximation with suitable choice of $h$ can be very accurate. If we consider larger values of $N$, we can observe that the approximate solution with $h=-1$ becomes more accurate than the other values of $h$ when $N \geq 8$. We then fix the values of $N$ and $h$, i.e., $N=10$ and $h=-1.1053$, and investigate the time evolution of the approximate analytical solutions for various $t$ and $\alpha$. The results are shown in Table 2. We observe that the 10-term approximate solutions with $h=-1.1053$ are very accurate. 


\begin{tabular}{rrrrrrr}
\hline$T$ & \multicolumn{2}{c}{$\alpha=1.2$} & \multicolumn{2}{c}{$\alpha=1.6$} & \multicolumn{2}{c}{$\alpha=2.0$} \\
\hline & Solution & Error & Solution & Error & Solution & Error \\
0.1 & 1.1200 & $2.6379 \mathrm{e}-12$ & 1.0354 & $1.3352 \mathrm{e}-12$ & 1.0100 & $1.3947 \mathrm{e}-12$ \\
0.2 & 1.2932 & $1.0809 \mathrm{e}-11$ & 1.1096 & $7.7398 \mathrm{e}-12$ & 1.0403 & $2.6135 \mathrm{e}-12$ \\
0.3 & 1.5111 & $3.6375 \mathrm{e}-11$ & 1.2150 & $7.4181 \mathrm{e}-12$ & 1.0914 & $2.4134 \mathrm{e}-12$ \\
0.4 & 1.7779 & $7.7804 \mathrm{e}-12$ & 1.3516 & $2.8288 \mathrm{e}-11$ & 1.1643 & $1.5979 \mathrm{e}-11$ \\
0.5 & 2.1011 & $1.9902 \mathrm{e}-10$ & 1.5211 & $6.9401 \mathrm{e}-11$ & 1.2606 & $2.9834 \mathrm{e}-11$ \\
0.6 & 2.4906 & $6.8443 \mathrm{e}-12$ & 1.7268 & $2.4791 \mathrm{e}-11$ & 1.3821 & $2.3717 \mathrm{e}-11$ \\
0.7 & 2.9584 & $9.4368 \mathrm{e}-10$ & 1.9733 & $1.7407 \mathrm{e}-10$ & 1.5313 & $2.6883 \mathrm{e}-11$ \\
0.8 & 3.5195 & $1.1823 \mathrm{e}-09$ & 2.2661 & $4.4433 \mathrm{e}-10$ & 1.7112 & $1.3323 \mathrm{e}-10$ \\
0.9 & 4.1916 & $2.1797 \mathrm{e}-09$ & 2.6120 & $4.8104 \mathrm{e}-10$ & 1.9254 & $2.7052 \mathrm{e}-10$ \\
1.0 & 4.9961 & $9.3082 \mathrm{e}-09$ & 3.0193 & $1.4490 \mathrm{e}-10$ & 2.1782 & $3.5943 \mathrm{e}-10$ \\
\hline
\end{tabular}

Table 2: $\left|U^{N}(x=0, T)-u_{\text {exact }}(x=0, T)\right|$ at $T=0.1,0.2, \ldots, 1$ and $\alpha=1.2,1.6$ and 2 when $N=10$ and $h=-1.1053$.

\subsection{Example 2}

We then consider the fourth-order time-fractional Boussinesq equation in two dimensions.

$$
\left\{\begin{array}{l}
{ }_{0} D_{t}^{\alpha} u=\beta_{1} u_{x x x x}+\beta_{2} u_{y y y y}+\gamma_{1} u_{x x}+\gamma_{2} u_{y y}+\theta_{1}\left(u^{2}\right)_{x x}+\theta_{2}\left(u^{2}\right)_{y y}-4\left(\theta_{1}+\theta_{2}\right) u^{2}, \quad-\infty<x, y<\infty, t>0, \\
u(x, y, 0)=e^{x+y}, \quad u_{t}(x, y, 0)=0 .
\end{array}\right.
$$

Here the solution $u$ is a function of $x, y$ and $t$. We let $\mathscr{L}(u)={ }_{0} D_{t}^{\alpha} u$ and $\mathscr{N}(u)={ }_{0} D_{t}^{\alpha} u-\beta_{1} u_{x x x x}-\beta_{2} u_{y y y y}-\gamma_{1} u_{x x}-\gamma_{2} u_{y y}-\theta_{1}\left(u^{2}\right) x_{x x}-$ $\theta_{2}\left(u^{2}\right)_{y y}+4\left(\theta_{1}+\theta_{2}\right) u^{2}$. Therefore,

$$
\begin{aligned}
{ }_{0} D_{t}^{\alpha} u_{1} & =h\left({ }_{0} D_{t}^{\alpha} u_{0}-\beta_{1}\left(u_{0}\right)_{x x x x}-\beta_{2}\left(u_{0}\right)_{y y y y}-\gamma_{1}\left(u_{0}\right)_{x x}-\gamma_{2}\left(u_{0}\right)_{y y}\right)-h\left(-\theta_{1}\left(u_{0}\right)_{x x}^{2}-\theta_{2}\left(u_{0}\right)_{y y}^{2}+4\left(\theta_{1}+\theta_{2}\right)\left(u_{0}\right)^{2}\right) \\
& =-h\left(\beta_{1}+\beta_{2}+\gamma_{1}+\gamma_{2}\right) e^{x+y} .
\end{aligned}
$$

Here we have chosen $u_{0}=e^{x+y}$. Therefore, we can obtain $u_{1}$ by applying the operator $J^{\alpha}$ on both sides of (3.9). That is,

$$
u_{1}(x, y, t)=-h \frac{\left(\beta_{1}+\beta_{2}+\gamma_{1}+\gamma_{2}\right) t^{\alpha}}{\Gamma(1+\alpha)} e^{x+y} .
$$

For $m \geq 2$, we get

$$
\begin{aligned}
{ }_{0} D_{t}^{\alpha}\left(u_{m}-u_{m-1}\right)= & h\left[{ }_{0} D_{t}^{\alpha} u_{m-1}-\beta_{1} \frac{\partial^{4} u_{m-1}}{\partial x^{4}}-\beta_{2} \frac{\partial^{4} u_{m-1}}{\partial y^{4}}-\gamma_{1} \frac{\partial^{2} u_{m-1}}{\partial x^{2}}-\gamma_{2} \frac{\partial^{2} u_{m-1}}{\partial y^{2}}\right]-h \theta_{1} \sum_{j=0}^{m-1} \frac{\partial^{2}\left(u_{j} u_{m-1-j}\right)}{\partial x^{2}} \\
& -h \theta_{2} \sum_{j=0}^{m-1} \frac{\partial^{2}\left(u_{j} u_{m-1-j}\right)}{\partial y^{2}}+4 h\left(\theta_{1}+\theta_{2}\right) \sum_{j=0}^{m-1} u_{j} u_{m-1-j} .
\end{aligned}
$$

We can show that when $m=2$, there is

$$
{ }_{0} D_{t}^{\alpha}\left(u_{2}-u_{1}\right)=h_{0} D_{t}^{\alpha} u_{1}+h^{2} \frac{\left(\beta_{1}+\beta_{2}+\gamma_{1}+\gamma_{2}\right)^{2} t^{\alpha}}{\Gamma(1+\alpha)} e^{x+y} .
$$

Thus, (3.10) and (3.12) lead to

$$
u_{2}(x, y, t)=-(1+h) h \frac{\left(\beta_{1}+\beta_{2}+\gamma_{1}+\gamma_{2}\right) t^{\alpha}}{\Gamma(1+\alpha)} e^{x+y}+h^{2} \frac{\left(\beta_{1}+\beta_{2}+\gamma_{1}+\gamma_{2}\right)^{2} t^{2 \alpha}}{\Gamma(1+2 \alpha)} e^{x+y} .
$$

Similarly, we can further derive the following formulations of $u_{m}(x, y, t)$ for $m=3$ :

$$
u_{3}(x, y, t)=-(1+h)^{2} h C_{1} t^{\alpha} e^{x+y}+2(1+h) h^{2} C_{2} t^{2 \alpha} e^{x+y}-h^{3} C_{3} t^{3 \alpha} e^{x+y},
$$

where

$$
C_{n}=\frac{\left(\beta_{1}+\beta_{2}+\gamma_{1}+\gamma_{2}\right)^{n}}{\Gamma(1+n \alpha)}, \quad n=1,2, \ldots
$$

In general, we can obtain

$$
u_{m}(x, y, t)=e^{x+y} \sum_{j=0}^{m-1}(-1)^{j+1}\left(\begin{array}{c}
m-1 \\
j
\end{array}\right) C_{j+1} h^{j+1}(1+h)^{m-1-j} t^{(j+1) \alpha},
$$

for $m \geq 1$. Thus the analytical solution is given by $u(x, y, t)=\sum_{m=0}^{\infty} u_{m}(x, y, t)$ where $u_{m}(x, y, t)$ is defined by equation (3.13). The 10-term approximate solution and its point-wise absolute error are plotted in Figure 3.1. It shows that the maximum absolute error occurs at $x=y=1$, and the overall error is at the magnitude of $10^{-8}$, which indicates the accuracy of the method. 

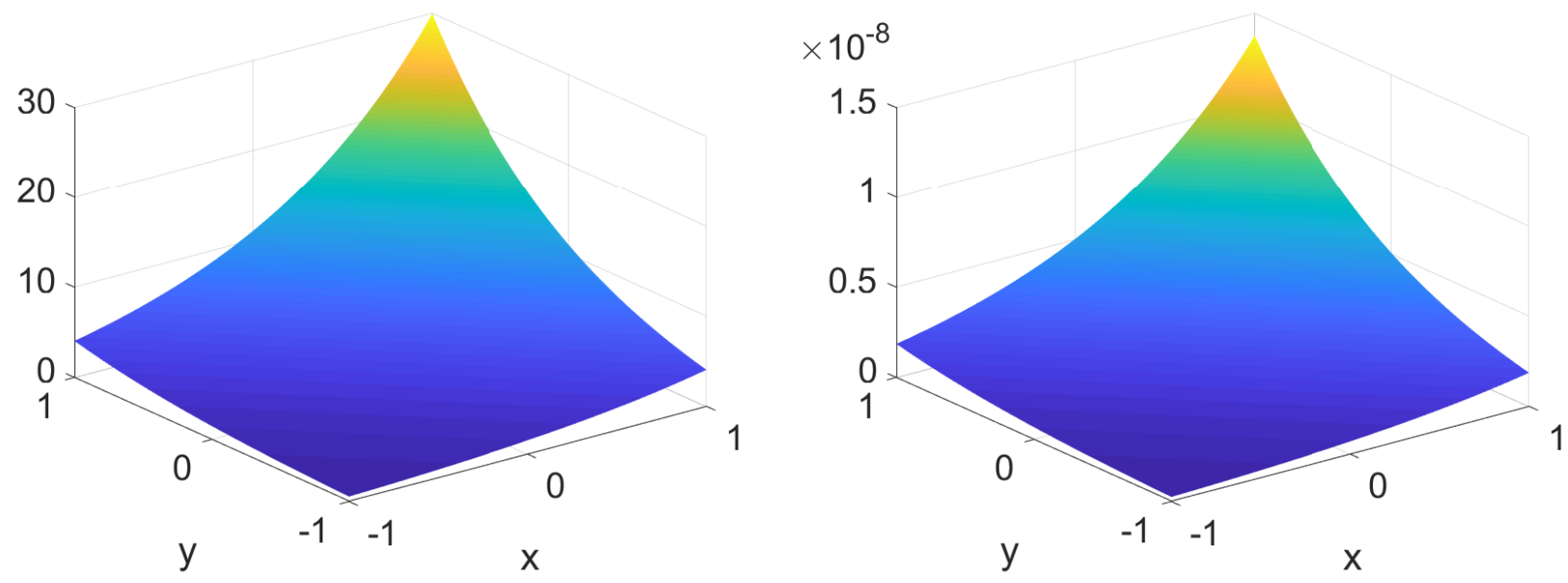

Figure 3.1: The 10-term approximate solution in example 2 and its absolute error. Here $\alpha=1.7, \beta_{1}=\gamma_{2}=1, \beta_{2}=\gamma_{1}=0.5$ and $T=1$. Left: the approximate solution at $T=1$. Right: the absolute error of the approximate solution at $T=1$.

\subsection{Example 3}

We then consider the time-fractional Boussinesq equation with the sixth-order spatial derivative:

$$
\left\{\begin{array}{l}
{ }_{0} D_{t}^{\alpha} u=u_{x x x x x x}+u_{x x x x}+u_{x x}+\theta\left(u^{2}\right)_{x x}-4 \theta u^{2}, \quad-\infty<x<\infty, t>0 \\
u(x, 0)=e^{x}, \quad u_{t}(x, 0)=0 .
\end{array}\right.
$$

From the process of homotopy analysis method, we have

$$
(1-q) \mathscr{L}\left(U(x, t ; q)-u_{0}(x, t)\right)=q h \mathscr{N}(U(x, t ; q)),
$$

where $\mathscr{L}(U(x, t ; q))={ }_{0} D_{t}^{\alpha} U, \mathscr{N}(U(x, t ; q))={ }_{0} D_{t}^{\alpha} U-U_{x x x x x x}-U_{x x x x}-U_{x x}-\theta\left(U^{2}\right){ }_{x x}+4 \theta U^{2}$ and $u_{0}(x, t)=e^{x}$. We then differentiate (3.14) with respect to $q$ for $m$ times, and let $q=0$ to get

$$
\begin{aligned}
& { }_{0} D_{t}^{\alpha}\left(u_{1}(x, t)\right)=h \mathscr{N}\left(u_{0}(x, t)\right), \\
& { }_{0} D_{t}^{\alpha}\left(u_{m}(x, t)\right)={ }_{0} D_{t}^{\alpha}\left(u_{m-1}(x, t)\right)+h \mathscr{R}\left(u_{1}, u_{2}, \ldots, u_{m-1}\right) .
\end{aligned}
$$

Here we have assumed that $U(x, t ; q)=\sum_{m=0}^{\infty} u_{m}(x, t) q^{m}$ is convergent for $q \in[0,1]$. The operator $\mathscr{R}$ is given by

$$
\mathscr{R}\left(u_{1}, u_{2}, \ldots, u_{m-1}\right)={ }_{0} D_{t}^{\alpha} u_{m-1}-\left(u_{m-1}\right)_{x x x x x}-\left(u_{m-1}\right)_{x x x x}-\left(u_{m-1}\right)_{x x}-\theta \sum_{j=0}^{m-1}\left(u_{j} u_{m-1-j}\right)_{x x}+4 \theta \sum_{j=0}^{m-1}\left(u_{j} u_{m-1-j}\right) .
$$

If we apply $J^{\alpha}$ to both sides of (3.15) and (3.16), we can obtain

$$
\begin{aligned}
& u_{1}(x, t)=h J^{\alpha} \mathscr{N}\left(u_{0}(x, t)\right), \\
& u_{m}(x, t)=u_{m-1}(x, t)+h J^{\alpha} \mathscr{R}\left(u_{1}, u_{2}, \ldots, u_{m-1}\right), \quad m \geq 2 .
\end{aligned}
$$

We can calculate the next three terms, i.e., $m=1,2$ and 3 , as follows

$$
\begin{aligned}
& u_{1}(x, t)=-3 h \frac{t^{\alpha} e^{x}}{\Gamma(1+\alpha)}, \\
& u_{2}(x, t)=-3(1+h) h \frac{t^{\alpha} e^{x}}{\Gamma(1+\alpha)}+9 h^{2} \frac{t^{2 \alpha} e^{x}}{\Gamma(1+2 \alpha)}, \\
& u_{3}(x, t)=-3(1+h)^{2} h \frac{t^{\alpha} e^{x}}{\Gamma(1+\alpha)}+18(1+h) h^{2} \frac{t^{2 \alpha} e^{x}}{\Gamma(1+2 \alpha)}-27 h^{3} \frac{t^{3 \alpha} e^{x}}{\Gamma(1+3 \alpha)} .
\end{aligned}
$$

To derive the general formulation of $u_{m}(x, t)$, we first let $u_{m}(x, t)$ be

$$
u_{m}(x, t)=\sum_{j=1}^{m} C_{m, j} h^{j}(1+h)^{m-j} \frac{t^{j \alpha} e^{x}}{\Gamma(1+j \alpha)},
$$

where $C_{m, j}$ is the undetermined coefficient in $u_{m}(x, t)$. From (3.20)-(3.22), we know that $C_{1,1}=C_{2,1}=C_{3,1}=-3, C_{2,2}=9, C_{3,2}=18$ and $C_{3,3}=-27$. Thus we only need to calculate $C_{m, j}$ for $m \geq 4$ and $j \in[1, m]$. Using equation (3.19), one can show that

$$
u_{m}(x, t)=(1+h) u_{m-1}(x, t)-3 h J^{\alpha} u_{m-1}(x, t) .
$$


We further apply (3.23) to (3.24) to get

$$
\begin{aligned}
u_{m}(x, t) & =\sum_{j=1}^{m-1} C_{m-1, j} h^{j}(1+h)^{m-j} \frac{t^{j \alpha} e^{x}}{\Gamma(1+j \alpha)}-\sum_{j=1}^{m-1} 3 C_{m-1, j} h^{j+1}(1+h)^{m-1-j} \frac{t^{(j+1) \alpha} e^{x}}{\Gamma(1+(j+1) \alpha)} \\
& =C_{m-1,1} h(1+h)^{m-1} \frac{t^{\alpha} e^{x}}{\Gamma(1+\alpha)}+\sum_{j=2}^{m-1}\left(C_{m-1, j}-3 C_{m-1, j-1}\right) h^{j}(1+h)^{m-j} \frac{t^{j \alpha} e^{x}}{\Gamma(1+j \alpha)}-3 C_{m-1, m-1} h^{m-1}(1+h) \frac{t^{m \alpha} e^{x}}{\Gamma(1+m \alpha)} .
\end{aligned}
$$

Therefore, we can derive the following difference equations for $m \geq 3$ :

$$
\left\{\begin{array}{l}
C_{m, 1}=C_{m-1,1}, \\
C_{m, j}=C_{m-1, j}-3 C_{m-1, j-1}, \quad \text { for } j \in[2, m-1], \\
C_{m, m}=-3 C_{m-1, m-1} .
\end{array}\right.
$$

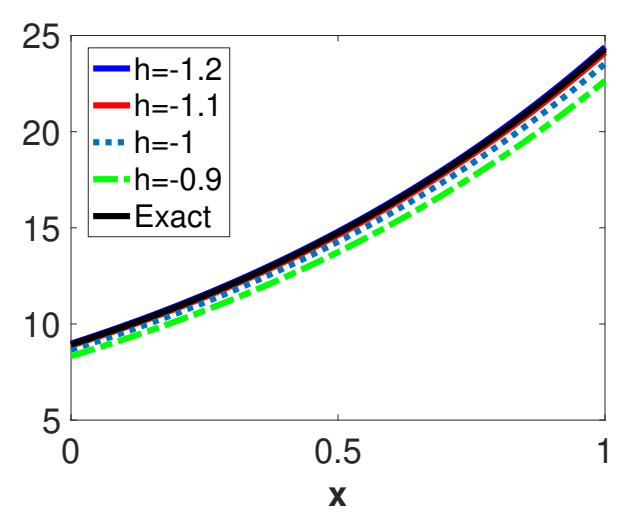

(a) $\alpha=1.25$

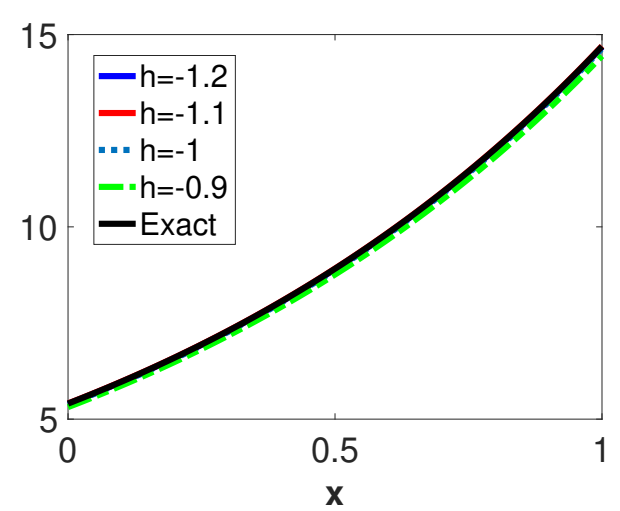

(c) $\alpha=1.5$

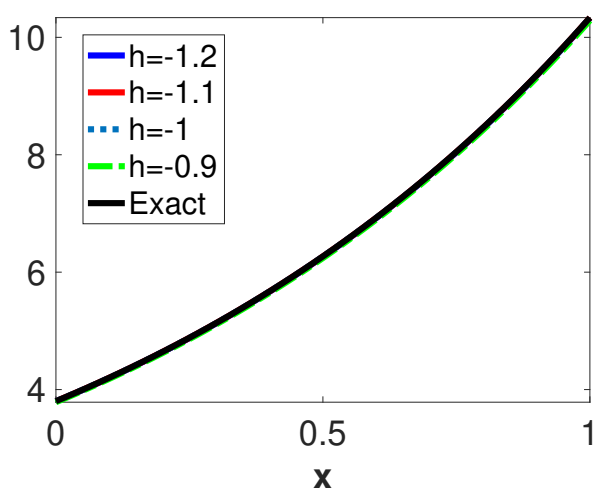

(e) $\alpha=1.75$

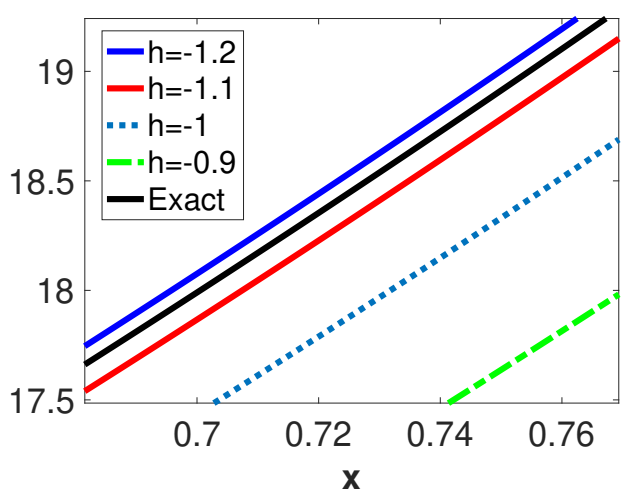

(b) $\alpha=1.25$ (zoomed-in plot)

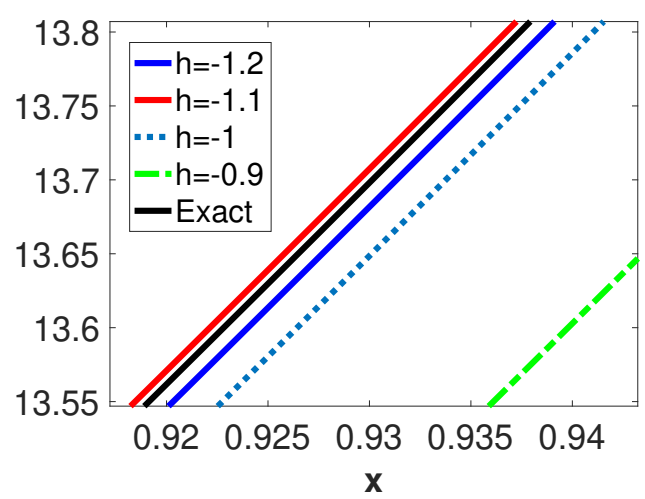

(d) $\alpha=1.5$ (zoomed-in plot)

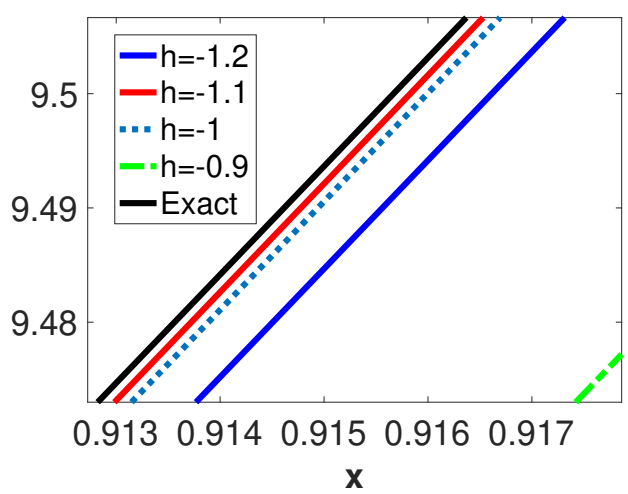

(f) $\alpha=1.75$ (zoomed-in plot)

Figure 3.2: Comparison of the 4-term approximate and exact solutions at the final time $T=1$ in example 3. Here $\alpha=1.25,1.5$ and 1.75. In (a)-(f), the blue, red, dark blue, green and black lines represent the approximate solutions with $h=-1.2,-1.1,-1,-0.9$ and the exact solution, respectively. (a): solutions when $\alpha=1.25$. (b): zoomed-in plot of solutions when $\alpha=1.25$. (c): solutions when $\alpha=1.5$. (d): zoomed-in plot of solutions when $\alpha=1.5$. (e): solutions when $\alpha=1.75$. (f): zoomed-in plot of solutions when $\alpha=1.75$. 
From the first equation in (3.25), we get $C_{m, 1}=C_{3,1}=-3$. The third equation in (3.25) leads to $C_{m, m}=(-3)^{m-2} C_{2,2}=(-3)^{m}$. For $j=2$ in the second equation of (3.25), we have $C_{m, 2}=C_{m-1,2}-3 C_{m-1,1}=C_{m-1,2}+9$. So we can derive $C_{m, 2}=C_{2,2}+9(m-2)=9(m-1)$. Similarly, we can also show that $C_{m, 3}=-\frac{27}{2}(m-1)(m-2)$ and $C_{m, 4}=\frac{27}{2}\left(m^{3}-3 m^{2}+2 m-18\right)$. The analytical formula of $C_{m, j}$ with $5 \leq j \leq m-1$ can be calculated in the similar manner, but we omit the detail here. The exact solution to the original problem is given by $\sum_{m=0}^{\infty} u_{m}(x, t)$

If we take $h=-1$, then according to equation (3.23), $u_{m}(x, t)=C_{m, m} h^{m} \frac{t^{m \alpha} e^{x}}{\Gamma(1+m \alpha)}=\frac{3^{m} t^{m \alpha} e^{x}}{\Gamma(1+m \alpha)}$. Therefore, the solution becomes $u(x, t)=$ $e^{x} E_{\alpha}\left(3 t^{\alpha}\right)$, where $E_{\alpha}$ is the Mittag-Leffler function defined in (2.4). For this example, we compute the 4-term approximate solutions with various values of $\alpha \in(1,2)$ and $h<0$, and plot the solutions in Figure 3.2. The top row in Figure 3.2 shows the 4-term approximate solutions for $h=-1.2,-1.1,-1$ and -0.9 when $\alpha=1.25$. We observe that the approximation solution with $h=-0.9$ is the least accurate solution, followed by the solution with $h=-1$. The 4-term approximation solution with $h=-1.2$ is slightly more accurate than that with $h=-1.1$. We can see that with appropriate choice of $h$, a 4-term approximation solution can be very accurate. When $\alpha=1.5$, similar results can be observed except that in this case the approximation solution with $h=-1.1$ is more accurate than that with $h=-1.2$. The bottom row in Figure 3.2 shows that when $\alpha=1.75, h=-1.1$ leads to the most accurate approximate solution. Based on the discussion above, we see that the parameter $h$ provides the flexibility of obtaining accurate solutions.

\section{Conclusion}

In this paper, we derive the analytical and the approximate analytical solutions of the one and two-dimensional time-fractional Boussinesq equation using the homotopy analysis method. The homotopy analysis method is a semi-analytical technique to solve the differential equations by representing the solution in the form of series with an auxiliary parameter $h$. We have demonstrated that the iterative method in [16] is a special case of the homotopy analysis method with $h=-1$. Numerical results show that the truncated $N$-term approximate solution with an appropriate value of $N$ can be used as an accurate approximation of the analytical solution. In particular, we find that $N=10$ with a suitable choice of $h$ leads to accurate approximate solutions when the final time is less than or equal to 1 , and we can obtain the approximate solutions with single-precision accuracy in the sense of $L^{\infty}$ norm. Moreover, the accuracy can be further improved by selecting the optimal value of $h$, which is the advantage of using this method over using the iterative method with $h=-1$.

\section{Acknowledgement}

The author would like to thank the editor and the anonymous reviewers for their constructive comments, which help to significantly improve the quality of the paper.

\section{References}

[1] A. A. Alikhanov, A new difference scheme for the time fractional diffusion equation, J. Comput. Phys., 280 (2015), 424-438.

[2] A. Atangana, D. Baleanu, New fractional derivatives with non-local and non-singular kernel theory and application to heat transfer model, Therm. Sci., 20 (2016), 763-769

[3] A. Bueno-Orovio, D. Kay, K. Burrage, Fourier spectral methods for fractional-in-space reaction-diffusion equations, BIT, 54 (2014), $937-954$.

[4] M. Caputo, M. Fabrizio, A new definition of fractional derivative without singular kernel, Prog. Frac. Differ. App., 1 (2015), 73-85.

[5] H. Hosseini, R. Ansari, New exact solutions of nonlinear conformable time-fractional Boussinesq equations using the modified Kudryashov method, Wave Random Complex, 27 (2017), 628-636.

[6] H. Jafari, N. Kadkhoda, D. Baleanu, Fractional Lie group method of the time-fractional Boussinesq equation, Nonlinear Dyn., 81 (2015), $1569-1574$.

[7] J. Jia, H. Wang, A fast finite volume method for conservative space-fractional diffusion equations in convex domains, J. Comput. Phys., 310 (2016), $63-84$.

[8] J. Jia, H. Wang, Fast finite difference methods for space-fractional diffusion equations with fractional derivative boundary conditions, J. Comput. Phys., 293 (2015), 359-369.

[9] A.A. Kilbas, H.M. Srivastava, J.J. Trujillo, Theory and applications of fractional differential equations, Elsevier, Amsterdam, 2006.

[10] S-J Liao, Beyond Perturbation: Introduction to the Homotopy Analysis Method, CRC, Boca Raton, 2004.

[11] Y. Lin, C. Xu, Finite difference/spectral approximations for the time-fractional diffusion equation, J. Comput. Phys., 225 (2007), 1533-1552.

[12] F. Mainardi, The fundamental solutions for the fractional diffusion-wave equation, Appl. Math. Lett., 9 (1996), $23-28$.

[13] F. Mainardi, Y. Luchko, G. Pagnini, The fundamental solution of the space-time fractional diffusion equation, Frac. Calc. Appl. Anal., 4 (2001), 153-192.

[14] M. Meerschaert, Tadjeran, Finite difference approximations for two-sided space-fractional partial differential equations, Appl. Numer. Math., 56 (2006), $80-90$.

[15] I. Podlubny, Fractional Differential Equations. An Introduction to Fractional Derivatives, Fractional Differential Equations, to Methods of their Solutions and Some of their Applications, Academic Press, San Diego, 1999.

[16] F. Xu, Y. Gao, W. Zhang, Construction of analytic solution for time-fractional Boussinesq equation using iterative method, Adv. Math. Phys., 2015, Article ID 506140, 7 pages.

[17] Q. Xu, J.S. Hesthaven, Discontinuous Galerkin method for fractional convection-diffusion equations, SIAM J. Numer. Anal., 52 (2014), 405-423.

[18] M. Yavuz, N. Özdemir, European vanilla option pricing model of fractional order without singular kernel, Fractal Fractional, 2 (2018), 1.

[19] M. Yavuz, N. Özdemir, On the solutions of fractional Cauchy problem featuring conformable derivative, Proceedings of ITM Web of Conferences, EDP Sciences, (2018), 01045.

[20] M. Yavuz, B. Yaşkiran, Homotopy methods for fractional linear/nonlinear differential equations with a local derivative operator, Balikesir Üniversitesi Fen Bilimleri Enstitüsü Dergisi, 20 (2018), 75-89.

[21] H. Yang, A new high-order method for the time-fractional diffusion equation with a source, J. Frac. Calc. Appl., 11 (2020), 111-129.

[22] H. Yang, J. Guo, J.-H. Jung, Schwartz duality of the Dirac delta function for the Chebyshev collocation approximation to the fractional advection equation, Appl. Math. Lett., 64 (2017), 205-212.

[23] F. Zeng, C. Li, F. Liu, I. Turner, The use of finite differencelelement approaches for solving the time-fractional subdiffusion equation, SIAM J. Sci. Comput., 35 (2013), A2976-A3000.

[24] H. Zhang, X. Jiang, M. Zhao, R. Zheng, Spectral method for solving the time fractional Boussinesq equation, Appl. Math. Lett., 85 (2018), 164-170. 